University of Wollongong

Research Online

Australian Institute for Innovative Materials -

Papers

Australian Institute for Innovative Materials

$1-1-2015$

Size-dependent chemical reactivity of silicon nanocrystals with water and oxygen

Melanie L. Mastronardi

University of Toronto

Kenneth K. Chen

University of Toronto

Kristine Liao

University of Toronto

Gilberto Casillas

University of Wollongong, gilberto@uow.edu.au

Geoffrey A. Ozin

University of Toronto

Follow this and additional works at: https://ro.uow.edu.au/aiimpapers

Part of the Engineering Commons, and the Physical Sciences and Mathematics Commons

Research Online is the open access institutional repository for the University of Wollongong. For further information contact the UOW Library: research-pubs@uow.edu.au 


\title{
Size-dependent chemical reactivity of silicon nanocrystals with water and oxygen
}

\author{
Abstract \\ A detailed investigation examines how the size of allylbenzene-capped silicon nanocrystals (ncSi:AB) \\ affects their chemical reactivity with gaseous $\mathrm{O}_{2}, \mathrm{H}_{2} \mathrm{O}$, and $\mathrm{O}_{2} / \mathrm{H}_{2} \mathrm{O}$ as probed by in situ luminescence \\ spectroscopy. Specifically, changes in the photoluminescence $(\mathrm{PL})$ of size-separated ncSi:AB are \\ monitored through alterations of their PL absolute quantum yield (AQY) as well as the wavelength and \\ intensity of their PL spectra over time. These experiments, conducted under both continuous and \\ intermittent illumination, help elucidate the roles of $\mathrm{O}_{2}, \mathrm{H}_{2} \mathrm{O}$, and mixtures of $\mathrm{O}_{2} / \mathrm{H}_{2} \mathrm{O}$, with respect to \\ oxidation of ncSi:AB as a function of their size, providing vital information for any perceived application in \\ advanced materials and biomedical devices.

\section{Keywords} \\ dependent, chemical, size, reactivity, oxygen, silicon, nanocrystals, water \\ Disciplines \\ Engineering | Physical Sciences and Mathematics

\section{Publication Details} \\ Mastronardi, M. L., Chen, K. K., Liao, K., Casillas, G. \& Ozin, G. A. (2015). Size-dependent chemical \\ reactivity of silicon nanocrystals with water and oxygen. The Journal of Physical Chemistry C: Energy \\ Conversion and Storage, Optical and Electronic Devices, Interfaces, Nanomaterials, and Hard Matter, 119 \\ (1), 826-834.
}




\title{
Size-Dependent Chemical Reactivity of Silicon Nanocrystals
}

\author{
Melanie L. Mastronardi, ${ }^{\dagger}$ Kenneth K. Chen, ${ }^{\dagger}$ Kristine Liao, ${ }^{\dagger}$ Gilberto Casillas, ${ }^{a}$ Geoffrey A. \\ Ozin ${ }^{+*}$
}

†Department of Chemistry, University of Toronto, 80 St. George Street, Toronto, Ontario, Canada, M5S 3H6
aUOW Electron Microscopy Centre, University of Wollongong, New South Wales 2500, Australia

KEYWORDS Silicon nanocrystals, size-dependent properties, photoluminescence, absolute quantum yield, surface oxidation, chemical reactivity

\begin{abstract}
The effects of exposure to dry and controlled humidity oxygenated atmospheres in the dark and light were examined in situ for monodisperse allylbenzene-capped silicon nanocrystals (ncSi:AB). Changes in photoluminescence (PL) intensity as measured by alterations of the PL absolute quantum yields (AQY) and blue shifting of PL spectra were measured over time under continuous and intermittent illumination to help elucidate the roles of $\mathrm{O}_{2}$ and $\mathrm{H}_{2} \mathrm{O}$ as well as UV irradiation in the oxidation of the ncSi surface. Size-dependent trends were observed for both PL blue shifting and changes in the PL AQY indicating that nanocrystal size plays an important role in how oxygen species react at the nanocrystal surface.
\end{abstract}

\section{Introduction}

Silicon nanocrystals (ncSi) were found to exhibit intense room temperature photoluminescence in 1990,1 and subsequent study has identified that they possess size-dependant optical and electronic properties, ${ }^{2 a-c}$ similar to other semiconductor nanocrystals composed of heavy metal materials. In particular, quantum and spatial confinement effects result in a widening of the electronic band gap and a blue shift in photoluminescence

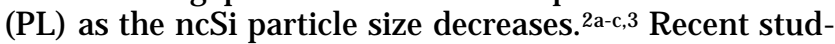
ies also report that ncSi show size-dependent PL absolute quantum yields (AQY). ${ }^{2 a, c, 4 a, b}$ These size-dependent properties as well as a wide PL range that spans the visible to near infrared region, ${ }^{2 c}$ have established ncSi as a promising candidate in optoelectronic and biological imaging applications. ${ }^{5 a-e}$

Currently, there are a variety of methods that exist for the synthesis of $n c S i$, including the annealing of $\mathrm{SiO}_{\mathrm{x}}$ powders followed by etching with $\mathrm{HF}^{6}$ plasma synthesis, ${ }^{7}$ and solution reduction of $\mathrm{SiCl}_{4}{ }^{8}$ Colloidal stability of $\mathrm{ncSi}$ is easily achieved through a hydrosilylation reaction between surface $\mathrm{Si}-\mathrm{H}$ groups and terminal alkenes. ${ }^{9 a-c}$ It is well established that surface capping with organic ligands helps to prevent unwanted oxidation of the nanocrystal surface; ${ }^{9 a, 10}$ however, overtime even capped ncSi have been shown to oxidize under ambient conditions. Exposure to air overtime, resulting in surface oxidation, is known to quench the PL of ncSi as well as blue shift its emission wavelength. $4 \mathrm{~b}, 11$ The blue shifting of PL has been attributed to the shrinking of the nanocrystalline core due from the formation of an oxide layer.3,12 The formation of dangling bonds and defect sites in the oxide layer create surface states in the band gap that effectively quench the PL. ${ }^{13 a, b}$
To date little is known regarding the role $\mathrm{O}_{2}$ and $\mathrm{H}_{2} \mathrm{O}$ plays in oxidation processes for ligand capped ncSi, but there are a variety of reports outlining different processes through which unfunctionalized or hydride-capped ncSi interact with $\mathrm{H}_{2} \mathrm{O}$ and $\mathrm{O}_{2}$. $^{13 a}$,14a-c Investigation of the oxidation of ncSi:H nanocrystals by electron spin resonance was reported to be consistent with the CabreraMott theory of oxidation in ambient-air. ${ }^{13 a}$ The process is proposed to involve an induction period where the preferential adsorption of $\mathrm{H}_{2} \mathrm{O}$ near surface silanol (Si-OH) groups aids the cleavage of an adjacent $\mathrm{Si}-\mathrm{Si}$ bond, which transfers an electron to an adsorbed $\mathrm{O}_{2}$ and attracts it to the cleaved bond. Reaction of the $\mathrm{O}_{2}$ at this site then leads to the formation of two Si-O-Si groups. Studies of bulk-Si surfaces indicated that the duration of the induction period is inversely proportional to humidity and density of $\mathrm{Si}-\mathrm{OH}$ groups. ${ }^{15 a, b}$

Hydride-terminated ncSi (ncSi:H) have been shown to be efficient photosensitizers of singlet oxygen due to their highly developed surfaces and long radiative lifetimes. ${ }^{14 a, b}$ This process quenches the PL of ncSi as energy is transferred from optically excited excitons to $\mathrm{O}_{2}$ molecules adsorbed on the ncSi surface and is most efficient at a PL wavelength of $760 \mathrm{~nm}(1.63 \mathrm{eV})$, which corresponds to the energy transition energy from the $\mathrm{O}_{2}$ triplet state to excited singlet state.14b PL quenching has been shown to be a reversible effect provided that the ncSi:H have not been exposed to $\mathrm{O}_{2}$ under prolonged illumination, which leads to an irreversible photooxidation process that degrades the PL permanently. ${ }^{14 c}$ Singlet $\mathrm{O}_{2}$ energy is not enough to break a Si-Si bond, therefore thermal energy is also required for the formation of Si-O$\mathrm{Si}$, which has been reported to form quicker at higher temperatures. ${ }^{14 c}$

While oxide formation at the ncSi surface tends to result in PL quenching, in some cases PL enhancement has 
also been observed; this is attributed to oxidationinduced passivation of dangling bonds.3,16a,b It has been proposed that the apparent discrepancy between different studies may result from variations in initial surface passivation and oxidation conditions. ${ }^{13 a}$ Overall the consensus is that interfacial defects and $\mathrm{Si}$ dangling bonds strongly influence the optoelectronic properties of ncSi, ${ }^{13 a}$ which means that understanding the surface structure of ncSi and how it oxidizes is of utmost importance for developing functional ncSi materials, particularly for colloidally-stable ligand-capped ncSi of varying size.

In order to probe the effects of oxidation on different sizes of organic capped $\mathrm{ncSi}$, we report the results of chemical reactivity studies performed on allybenzenecapped ncSi that have been size-separated into relatively monodisperse fractions using size-selective precipitation. ${ }^{2 a}$

\section{Experimental}

Reagents and Materials. Trichlorosilane ( $\mathrm{HSiCl} 3$, 99\%) was purchased from Sigma Aldrich, stored in a refrigerator, and used as received. Hydrofluoric acid (ACS reagent 48\%, Sigma Aldrich), ethanol (anhydrous, Commercial Alcohols), methanol (ACS reagent, Sigma Aldrich), toluene (anhydrous, Sigma Aldrich), isopropanol (ACS, EMD), potassium hydroxide (technical grade, $\mathrm{ACF}$ ), hexanes (ACS reagent, Caledon Laboratories), and phenyltriethoxysilane (98\%, Aldrich) were used as received. Allylbenzene (98\%, Aldrich) was filtered through activated neutral alumina (type WN-3, Sigma Aldrich) and a $0.2 \mu \mathrm{m}$ PTFE syringe filter before use to remove any peroxide impurities. ${ }^{17}$ Argon compressed gas (ultrahigh purity, Praxair) and 49.9\% oxygen balance argon compressed gas (certified standard, Praxair) were purged through deionized water or Drierite.

Synthesis of Size-Separated ncSi:AB. Sizeseparated allylbenzene-capped ncSi were synthesized using a previously reported method with some modifications. $^{2 a}$ This method involves the high temperature thermal processing of sol-gel hydridosilicate $\left(\mathrm{HSiO}_{1.5}\right)$ glasses derived from trichlorosilane $\left(\mathrm{HSiCl}_{3}\right)$, followed by etching with $\mathrm{HF}$ to liberate the ncSi from the encapsulating $\mathrm{SiO}_{2}$ matrix. The hydride-terminated ncSi were functionalized with allylbenzene groups using a thermallyinitiated hydrosilylation reaction in neat allylbenzene to produce a stable, concentrated colloidal dispersion. The ncSi that were too large to be stabilized with allylbenzene caps were removed from the reaction mixture by centrifugation and the ncSi:AB were isolated from the excess un-reacted allylbenzene by vacuum distillation followed by heating under vacuum at $155^{\circ} \mathrm{C}$ for 5.5 hours to removed excess alkenes. ${ }^{18}$ The purified polydisperse ncSi:AB were re-dispersed in toluene, resulting in a clear orange solution that displayed bright orange-red photoluminescence (PL) under photoexcitation $\left(\lambda_{\text {exc }}=365\right.$ $\mathrm{nm})$. Size-selective precipitation was performed on the toluene dispersion of polydisperse ncSi:AB, using methanol as the anti-solvent to isolate fractions of relatively monodisperse ncSi:AB. Following each addition of methanol, the cloudy solution was briefly sonicated then centrifuged for $10 \mathrm{mins}$ at $6461 \mathrm{rpm}$. The clear supernatant was decanted and the solid was immediately redis- persed in toluene. In order to ensure sample purity, each fraction was precipitated a second time. Immediately after the supernatant was decanted after the second precipitation the solid precipitate was transferred into a glove box in order to minimize surface oxidation from exposure to air. In the glove box, the solid precipitate for each fraction was dispersed in anhydrous toluene and stored until used.

Preparation of ncSi:AB Films. Glass slide substrates were treated in a mixture of $\mathrm{KOH}$ and isopropanol (sonicated for one hour and left overnight), then rinsed with distilled water and dried in an oven. Dried substrates were then coated with phenyltriethoxysilane and heated in an oven at around $120^{\circ} \mathrm{C}$ for 3 days. The substrates were then rinsed with hexanes, dried and transferred to a glove box. 40ul of a toluene dispersion of ncSi:AB was then drop-coated onto the hydrophobic glass substrates, and allowed to dry.

Characterization. Scanning transmission electron microscopy (STEM) images were acquired in a probecorrected J EOL ARM200F operated at $80 \mathrm{kV}$ equipped with a cold field emission gun and a high resolution polepiece. Middle angle annular dark field (MAADF) images were acquired with 45 and 180 mrad inner and outer collection angles respectively, while bright field images used 11 mrad collection angles. Both images were recorded with a dwell time of $38 \mu \mathrm{s}$, and convergence semiangle of 25 mrad resulting in a probe current of $40 \mathrm{pA}$. Samples were drop-cast onto Graphene-Cu grids (Graphene Supermarket) to ensure an ultra-thin support film of less than $2 \mathrm{~nm}$. At least 500 particles were measured by hand to determine the average particle diameter and standard deviation values of the ncSi:AB from microscopy images. The photoluminescence of the ncSi:AB fractions was obtained using the absolute quantum yield setup described below. The absolute quantum yield was determined using a previously reported integrating sphere method. ${ }^{19}$ Samples were excited in an integrating sphere (Gigahertz Optik, custom made) with light from a 365nm LED (Thorlabs M365L2), and photoluminescence was collected by a $1 \mathrm{~mm}$ diameter optical fibre (Ocean Optics) and detected by an Ocean Optics Maya 2000 spectrometer. The continuous absolute quantum yield was measured by taking appropriate ratios of the excitation and emission peak areas of spectra recorded for the empty sphere and sample holder, and the excitation focused directly onto the sample. ${ }^{20}$ The accuracy of the system was verified against literature values for rhodamine 6G and MEH-PPV. Solution samples were measured in cuvettes that were filled and sealed in the glove box. Film samples were sealed into a custom made sample holder in the glove box that was then inserted into the integrating sphere. The continuous AQY of each film sample was measured over a period of 300 seconds (5 minutes), and intermitted illumination was achieved by blocking the LED light from hitting the sample over short periods of time throughout the 5 minute measurement. During each measurement, the film samples were exposed to different atmospheres. Dry argon and dry $50 \%$ oxygen in argon were created by flowing gas through a tube containing Drierite. Wet argon and wet $50 \%$ oxygen in argon were created by flowing gas through a bubbler filled with deionized water. The hu- 
midity of the atmospheres was monitored using a relative humidity probe (RH-USB, Omega Engineering, Inc.). All AQY and PL film experiments were conducted in duplicate, and the extracted AQY and PL values were averaged wherever possible. PL spectra were smoothed and baseline corrected using Origin Pro 8 software. Fourier transform infrared (FTIR) spectroscopy was performed on a Perkin Elmer Spectrum One FTIR spectrometer and peak areas were determined using Spectrum software. For pristine samples, films of ncSi: $\mathrm{AB}$ were drop-cast onto an IR transparent $\mathrm{KBr}$ substrate in the glove box, covered with a second $\mathrm{KBr}$ substrate, and sealed with Teflon tape. For samples exposed to different atmospheres, films of $\mathrm{ncSi}: \mathrm{AB}$ were drop-cast onto $6 \mathrm{~mm}$ by $1 \mathrm{~mm} \mathrm{KBr}$ plates, loaded into the resealable continuous AQY sample holder in the glove box, and exposed to the gas flow as above, under illumination in the integrating sphere or in the dark. The plates were then removed and run immediately under ambient conditions. X-ray photoelectron spectra (XPS) were obtained using a Thermo Scientific Theta Probe utilizing monochromatic $\mathrm{Al} \mathrm{K}$ a radiation, and spectra were calibrated to the C1s emission arising from adventitious hydrocarbons $(284.8 \mathrm{eV})$. The high-resolution $\mathrm{Si} 2 \mathrm{p}$ region of the XPS was fit for the dominant $\mathrm{Si}$ species $\left(\mathrm{Si}^{0}, \mathrm{Si}^{+1}, \mathrm{Si}^{+2}\right.$, $\mathrm{Si}^{+3}$, and $\mathrm{Si}^{+4}$ ). The atomic \% determined from the area of the C1s, O1s and Si2 $\mathrm{p}^{0}$ peaks were used to compare the extent of surface oxidation of pristine and air exposed samples of varying sizes.

\section{Results and Discussion}

The polydisperse ensemble and select size-separated ncSi:AB samples were characterized by scanning transmission electron microscopy (STEM). Particle size analysis confirms the expected decreasing particle size with increasing size-selective precipitation step (SI Figure 1). The average diameter values were measured as $2.79 \pm$ $1.7 \mathrm{~nm}$ for the ensemble, $2.22 \pm 0.5 \mathrm{~nm}$ for fraction 11 , $2.14 \pm 0.4 \mathrm{~nm}$ for fraction $13,2.01 \pm 0.4 \mathrm{~nm}$ for fraction 16 , and $1.81 \pm 0.4 \mathrm{~nm}$ for fraction 18. All fractions contain some degree of crystallinity, with crystal lattice lines appearing in the images of all samples examined. However, lattice fringes are not observed for all particles, which may be due to random orientation of the particles and beam damage that causes the particles to amorphise before an image can be acquired. It is not possible to say if all particles were crystalline to begin with, but there are certainly some crystalline particles observed for all fractions including fraction 18, which contains the smallest particles (SI Figure 2).
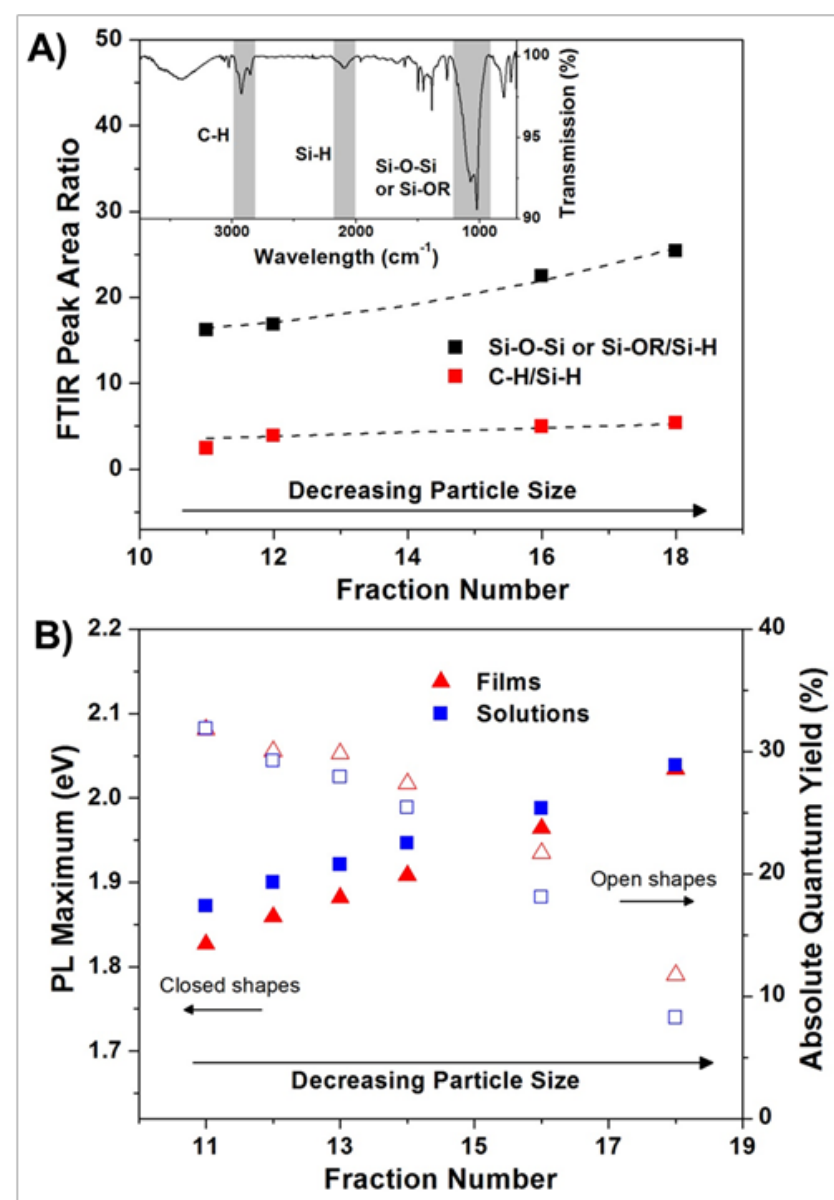

Figure 1. Characterization of Size-Separated ncSi:AB. A) Ratios of FTIR peak areas plotted for select size-separated ncSi:AB fractions. Inset, denotes peak area regions examined on the FTIR spectrum of fraction 11 . B) Photoluminescence (PL) maximum and PL absolute quantum yield (AQY) plotted versus fraction number for both film and solution samples show the variation of values for each fraction in different forms. The difference in PL maximum value decreases while the difference in AQY increases with decreasing size.

Select pristine samples of size-separated ncSi:AB were analyzed using Fourier Transform infrared (FTIR) spectroscopy. The FTIR spectrum shown for fraction 11 (Figure 1A, inset) shows the characteristic stretching modes associated with allybenzene capped ncSi, consistent with previous work. ${ }^{2 a}$ To compare the surface species present for fractions of different size, ratios of the areas associated with $\mathrm{Si}-\mathrm{H}, \mathrm{C}-\mathrm{H}$, and $\mathrm{Si}-\mathrm{O}-\mathrm{Si}$ or $\mathrm{Si}-\mathrm{OR}$ stretching modes are plotted in Figure 1A. The ratio of $\mathrm{C}-\mathrm{H}$ to $\mathrm{Si}-\mathrm{H}$ areas increases slightly with decreasing size, indicating that there is a higher proportion of allylbenzene ligands to $\mathrm{Si}-\mathrm{H}$ species at the surface of smaller $\mathrm{ncSi}: \mathrm{AB}$. This can be explained by the increasing surface curvature of particles with decreasing size. Complete surface coverage of ncSi with any alkyl ligand is not possible due to the disparity between the diameter of an alkyl chain carbon $(4.2 \AA)$ and the spacing between silicon atoms on the Si surface (3.85 $\AA$ for 111). ${ }^{21}$ Ligand cone models have been reported and used to estimate the surface coverage of ligands on nanoparticle surfaces. ${ }^{22}$ For allylbenzene, which has a higher ligand cone angle compared to a linear alkyl group, a higher surface curvature 
will allow more room for ligands to spread out from the nanocrystal surface, allowing more $\mathrm{Si}-\mathrm{H}$ species to be converted to $\mathrm{Si}-\mathrm{AB}$ with decreasing particle size. The presence of adventitious surface oxidation has been reported in previous syntheses of organic capped $\mathrm{ncSi}, 5 \mathrm{c}, 23$ and the results here show the ratio of the peak associated with $\mathrm{Si}-\mathrm{O}-\mathrm{Si}$ or $\mathrm{Si}-\mathrm{OR}$ to $\mathrm{Si}-\mathrm{H}$ increasing with decreasing size, indicating that a greater proportion of oxide species exist at the ncSi:AB surface of smaller particles. This size-dependent adventitious surface oxidation trend is also observed in comparing the atomic \% of core $\mathrm{Si}, \mathrm{O}$ and $\mathrm{C}$ species determined by XPS for particles of varying size (SI Figure 3A). The $\mathrm{Si}-\mathrm{H}$ species that remain at the $n c S i: A B$ surface likely react with residual oxygen dissolved in the toluene or methanol itself, which was used as the anti-solvent in the size-separation step. The sizedependence of the adventitious surface oxidation can be explained by the increasing spacing between the ligand tails that make it easier for oxide species to reach the surface with increasing curvature, ${ }^{24}$ as well as the fact that smaller sized particles were exposed to methanol for longer time periods due to the nature of the sizeseparation process. Despite the presence of oxide surface species, there is still a significant peak associated with Si$\mathrm{H}$ for all fractions, indicating the presence of sites with which oxygen species in the oxygen and water rich environments used can react for this chemical reactivity study.

The PL spectra and PL AQY were measured for select pristine fractions of ncSi:AB both dispersed in toluene and drop-coated onto treated hydrophobic glass slide substrates. The AQY shows a decreasing trend with decreasing particle size for both film and solution samples (Figure 1B), which is consistent with previous reports of similarly sized ncSi. ${ }^{2 a, 4 b}$ In previous work, the AQY decrease has been attributed to a decrease in ncSi core crystallinity with decreasing particle size, as well as an increase in the competing non-radiative relaxation surface trap and defect pathways that become more prevalent with decreasing $n c S i$ size. ${ }^{2 a}$ The microscopy and FTIR results presented here tend to favor the later explanation, since some crystallinity is observed for even the smallest particles imaged and the ratio of oxide species, likely to contribute to surface traps and defect sites, is shown to increase with decreasing particle size.

Consistent with the effects of quantum confinement, the PL maximum blue shifts with decreasing particle size for both film and solution samples (Figure 1B). ${ }^{4 a, 13 b}$ A slight red shift in PL peak emission for film versus solutions samples is attributed to greater particle interactions in the solid state film samples, resulting in interparticle coupling or efficient energy transfer from the smaller particles to the larger particles. ${ }^{25 a, b}$
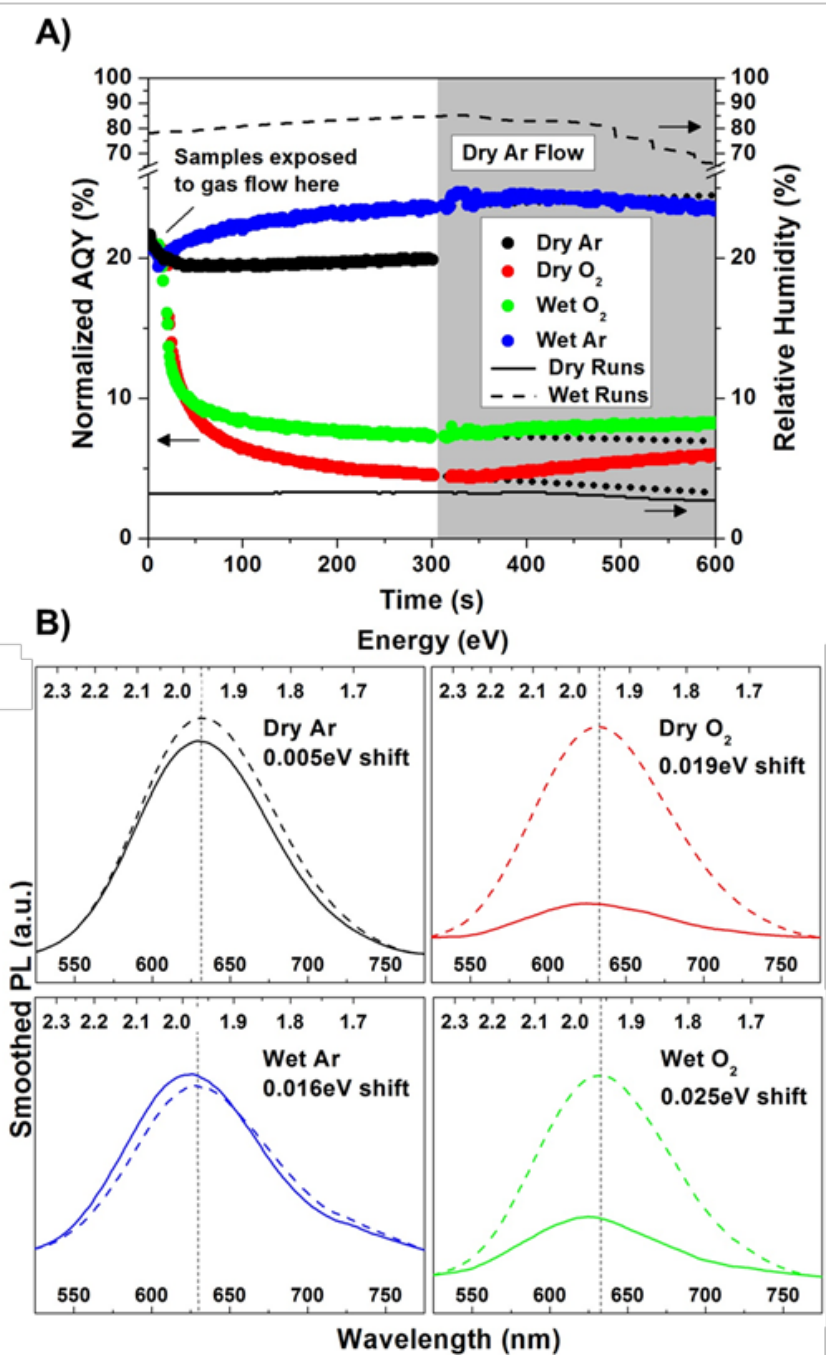

Figure 2. Continuous Absolute Quantum Yield Measurements. A) Photoluminescence (PL) absolute quantum yield (AQY) curves for fraction 16 measured under different atmospheres, showing representative relative humidity curves for dry and wet atmospheres. The AQY curves have been normalized to the average initial AQY value. After 300s the atmospheres containing $\mathrm{H}_{2} \mathrm{O}$ and/ or $\mathrm{O}_{2}$ were purged for 300s with dry Ar flow through the system. The dotted lines shown following the switch to dry Ar are guides to help visualize the changes in AQY. B) PL spectra for fraction 16 measured before (dashed line) and after exposure (solid line) to each atmospheres for 300s. PL spectra have been smoothed for clarity. PL blue shift from the initial value for each atmosphere is denoted in $\mathrm{eV}$.

In order to probe the effect different oxygen species have on the optical properties of ncSi:AB, the AQY was measured continuously while film samples of select fractions were exposed to different atmospheres. Initially each sample was prepared and sealed under nitrogen; the samples were then exposed to one of the following atmospheres: dry argon, wet argon, dry 50\% oxygen in argon (referred to as dry $\mathrm{O}_{2}$ ), and wet $50 \%$ oxygen in argon (referred to as wet $\mathrm{O}_{2}$ ). PL spectra were measured initially and after exposure to each atmosphere for 300s. To help elucidate the extent of non-chemical quenching observed select films were subsequently purged with dry Ar following exposure to $\mathrm{O}_{2}$ and $\mathrm{H}_{2} \mathrm{O}$ containing atmospheres. The relative humidity was monitored during all 
continuous AQY measurements using a relative humidity sensor. In addition to the continuous AQY measurements outlined above, film samples were run under all atmospheres with intermittent illumination to study the effect of light on the reactivity of $\mathrm{H}_{2} \mathrm{O}$ and/ or $\mathrm{O}_{2}$ species at the $\mathrm{nCSi}: \mathrm{AB}$ surface.

AQY curves and relative humidity curves representative of wet and dry conditions under continuous illumination are shown for fraction 16 in Figure 2A and the corresponding PL spectra are shown in Figure 2B. In the analysis of Figure 2, it is useful to assume a baseline with the results of inert dry Ar exposure at 300s where the signals have stabilized in order to measure and understand the effects of $\mathrm{H}_{2} \mathrm{O}$ and $\mathrm{O}_{2}$. Despite efforts to purge and eliminate air from the testing apparatus, it is likely that some residual ambient air remained; this is demonstrated by a slight decrease in AQY (from 22 to $20 \%)$ and slight blue shift in PL maximum $(0.005 \mathrm{eV})$ observed for the P16 dry Ar sample. Recent work suggest that exposure to UV light under inert conditions results in a decrease in AQY due to non-oxidative photobleaching, ${ }^{26}$ which may also be contributing to the decrease in AQY we observe. However, the work presented here was performed on much a shorter timescale and in our case we observe a blue shift in PL indicating that oxidation from residual air is the dominant effect at play.

Upon exposure to atmospheres containing $\mathrm{O}_{2}$ (both wet and dry) a significant decrease in $\mathrm{AQY}$ was observed. At 300 s, AQY values were $7 \%$ and $4.5 \%$ for samples exposed to wet $\mathrm{O}_{2}$ and dry $\mathrm{O}_{2}$, respectively. Additionally, we see that the PL has blue-shifted for both dry $(0.019 \mathrm{eV})$ and wet $\mathrm{O}_{2}(0.025 \mathrm{eV})$. Since blue shifting of ncSi emission energy has been attributed to shrinking of the nanocrystalline core due to the formation of an oxide layer for ncSi exposed to air overtime, it can be inferred that some form of irreversible oxidation is occurring for samples in all atmospheres. This oxidation would also result in some PL quenching (decrease in AQY) due to the emergence of intermediate states caused by the formation of dangling bonds and defect sites. $13 \mathrm{a}, \mathrm{b}$ The large AQY decrease and PL blue shift observed for both samples exposed to $\mathrm{O}_{2}$ indicate that surface oxidation is responsible for a large extent of the optoelectronic changes.

Purging with dry Ar beginning at 300s, results in an increase of the AQY of dry $\mathrm{O}_{2}$ treated ncSi: $\mathrm{AB}$ from 4.5 to $6 \%$ and an increase from 7 to $8 \%$ for wet $\mathrm{O}_{2}$ treated samples by 600 s. Based on this increase in AQY we propose that the AQY decrease observed at 300s is due to a combination of defect states formed from irreversible surface oxidation as well as photosensitization, which would give some reversible PL quenching. Photosensitization in this case describes the process where photogenerated singlet oxygen molecules cause photodegradation in silicon. ${ }^{14 c}$ Molecular oxygen adsorbed to ncSi surfaces act as a competing mechanism through which photogenerated excitons within the ncSi may transfer its energy, leading to the generation of singlet oxygen. The reactive photogenerated singlet oxygen may then react with the ncSi surface, leading to irreversible photooxidation. It is likely that the dry Ar purge at 300s removed some of the adsorbed oxygen to reduce its depressing effect on the AQY.

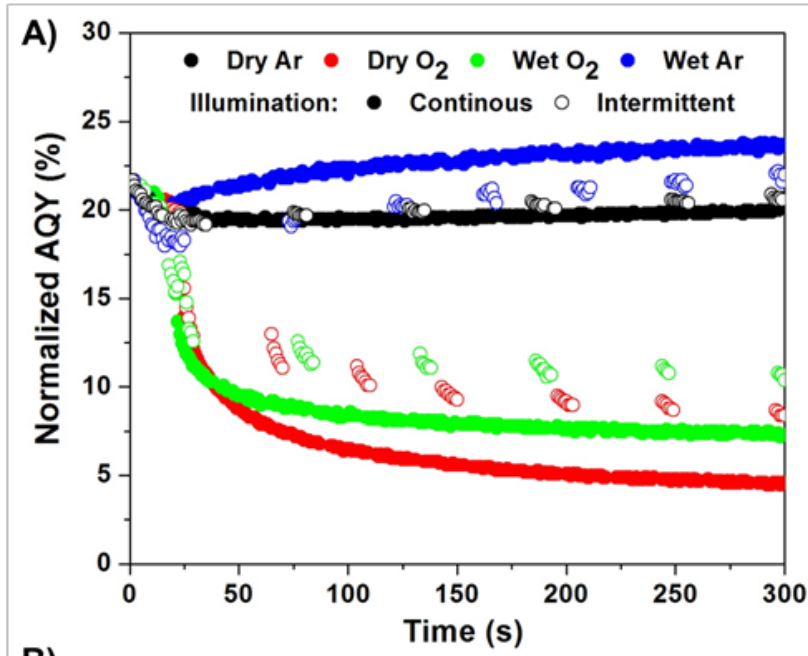

B)

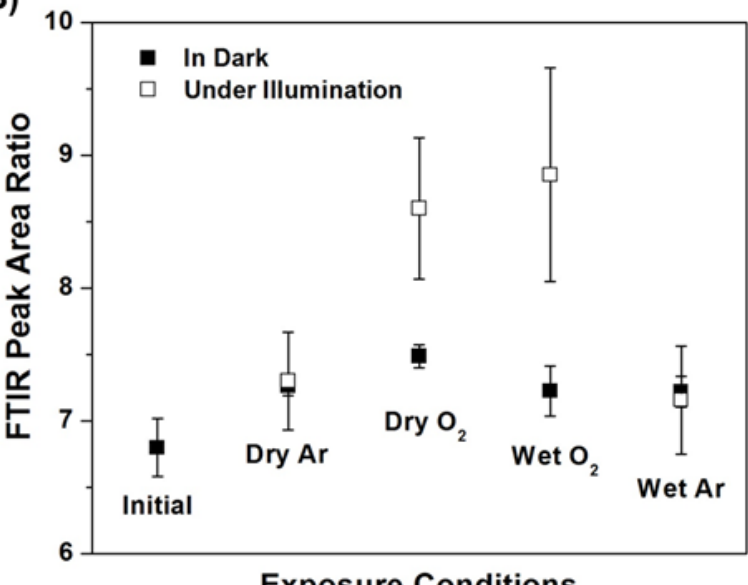

Figure 3. A) Absolute quantum yield (AQY) curves for fraction 16 measured under different atmospheres with continuous and intermittent illumination. B) Ratio of the Si-O$\mathrm{Si} / \mathrm{Si}-\mathrm{OR}$ to $\mathrm{Si}-\mathrm{H}$ peak areas for fraction 10 initially and after exposure to different conditions under illumination and in the dark for 300s.

The contribution of $\mathrm{H}_{2} \mathrm{O}$ on irreversible surface oxidation and photosensitization is difficult to quantify, but by comparing the results of wet and dry $\mathrm{O}_{2}$ exposure in Figure 2, we begin to see a few trends. The larger initial AQY decrease and lower AQY increase with dry Ar purging for wet $\mathrm{O}_{2}$ compared to dry $\mathrm{O}_{2}$ (Figure 2A), suggests that a greater extent of irreversible surface oxidation versus photosensitization is contributing to the AQY changes in the wet $\mathrm{O}_{2}$ atmosphere. Additionally, we observe a larger blue shift for wet $\mathrm{O}_{2}(0.025 \mathrm{eV})$ compared to dry $\mathrm{O}_{2}(0.019 \mathrm{eV})$ (Figure 2B). These results are consistent with reports where $\mathrm{H}_{2} \mathrm{O}$ passivates dangling bonds generated by oxidation by forming $\mathrm{Si}-\mathrm{OH}$ groups, ${ }^{13 a}$ and higher humidity with higher density of Si$\mathrm{OH}$ groups helps shorten the induction period for surface oxidation of $\mathrm{Si}$. ${ }^{15 a, b}$

In Figure 2, ncSi:AB exposed to wet Ar shows an increase in AQY up to $4 \%$ above the dry Ar baseline, with a PL blue shift $(0.016 \mathrm{eV})$ that is 3 times greater than that of ncSi:AB exposed dry Ar after 300s. The larger blue shift compared to dry Ar is likely the result of residual air in the system, as previously described, that in the presence of $\mathrm{H}_{2} \mathrm{O}$ would be much more likely to oxidize the 
surface. It has been proposed that physisorbed $\mathrm{H}_{2} \mathrm{O}$ may be responsible for air-induced PL degradation of $\mathrm{ncSi} ; 26$ however, the results presented here indicate that this is not the case when ncSi:AB is exposed to pure $\mathrm{H}_{2} \mathrm{O}$. The increase in AQY is likely due to $\mathrm{H}_{2} \mathrm{O}$ passivation of defects states and dangling bonds present on the ncSi:AB surface. ${ }^{13 a}$ With dry Ar purging after 300s, the AQY continues to increase slightly then begins to decline after about 450s. Assuming the interaction between ncSi:AB and pure $\mathrm{H}_{2} \mathrm{O}$ to passivate defects takes place as both a chemical reaction and physical adsorption, removing some of the excess $\mathrm{H}_{2} \mathrm{O}$ with dry $\mathrm{Ar}$ purging could result in reducing the passivation effect thereby decreasing the AQY.

Since a source of photogenerated excitons is a key to photosensitization of $\mathrm{O}_{2}$ and subsequent photooxidation, an experiment with intermittent, rather than continuous illumination and AQY measurement was conducted as shown in Figure 3A. The results show that when intermittent illumination is used the overall effect of each atmosphere on the AQY is not as severe as with continuous illumination, indicating the strong role of light in the interaction between $\mathrm{H}_{2} \mathrm{O}$ and $\mathrm{O}_{2}$ with the ncSi:AB surface. At 300s exposure, despite the presence of some residual air, the AQY for ncSi:AB exposed to dry Ar remains stable at approximately $20.5 \%$. Intermittent illumination results in minimizing the AQY decrease for ncSi:AB in dry $\mathrm{O}_{2}$, reaching only about $8.5 \%$ instead of $4.5 \%$ under continuous illumination after $300 \mathrm{~s}$. In wet $\mathrm{O}_{2}$ the ncSi:AB AQY decrease is minimized as well but to a slightly lower extent, reaching only about $10 \%$ instead of $7 \%$ under continuous illumination. The lower decreases observed for $\mathrm{O}_{2}$ exposed samples are consistent with previously reported work of polydisperse ncSi exposed to air and with and without UV illumination. ${ }^{26}$ The ncSi:AB exposed to wet Ar increases to about $22 \%$, which is slightly lower than the value of $24 \%$ achieved under continuous illumination, indicating that the passivation of surface defects is promoted with additional energy.

To confirm the effect of photosensitization on surface oxidation and ultimately the AQY and PL maximum, film samples of fraction 10 drop-coated on $\mathrm{KBr}$ windows were subject to the four atmospheres and FTIR measurement of the samples were taken. The area ratios of peaks assigned to $\mathrm{Si}-\mathrm{H}$ stretch $\left(2100 \mathrm{~cm}^{-1}\right)$ and $\mathrm{Si}-\mathrm{O}-\mathrm{Si} / \mathrm{Si}-\mathrm{OR}$ stretch $\left(1090 \mathrm{~cm}^{-1}\right)$ were plotted in Figure 3B. Compared to the initial value, all samples show a slightly higher ratio due to oxidation attributed to residual air prior to the experiment. While there is almost no difference in the ratio for wet and dry Ar, both dry and wet $\mathrm{O}_{2}$ exposed samples show a significantly higher ratio under continuous illumination, which is evidence of the extent photooxidation contributes to the formation of $\mathrm{Si}-\mathrm{O}-\mathrm{Si}$ species and the optoelectronic changes observed. Unfortunately, the extent of $\mathrm{Si}-\mathrm{OH}$ on the $\mathrm{ncSi}: \mathrm{AB}$ surface cannot be probed using FTIR under wet and dry conditions as the $\mathrm{Si}-\mathrm{OH}$ peak overlaps with the peak associated with adsorbed $\mathrm{H}_{2} \mathrm{O}$ in the region between 3200$3600 \mathrm{~cm}^{-1}$.

A)
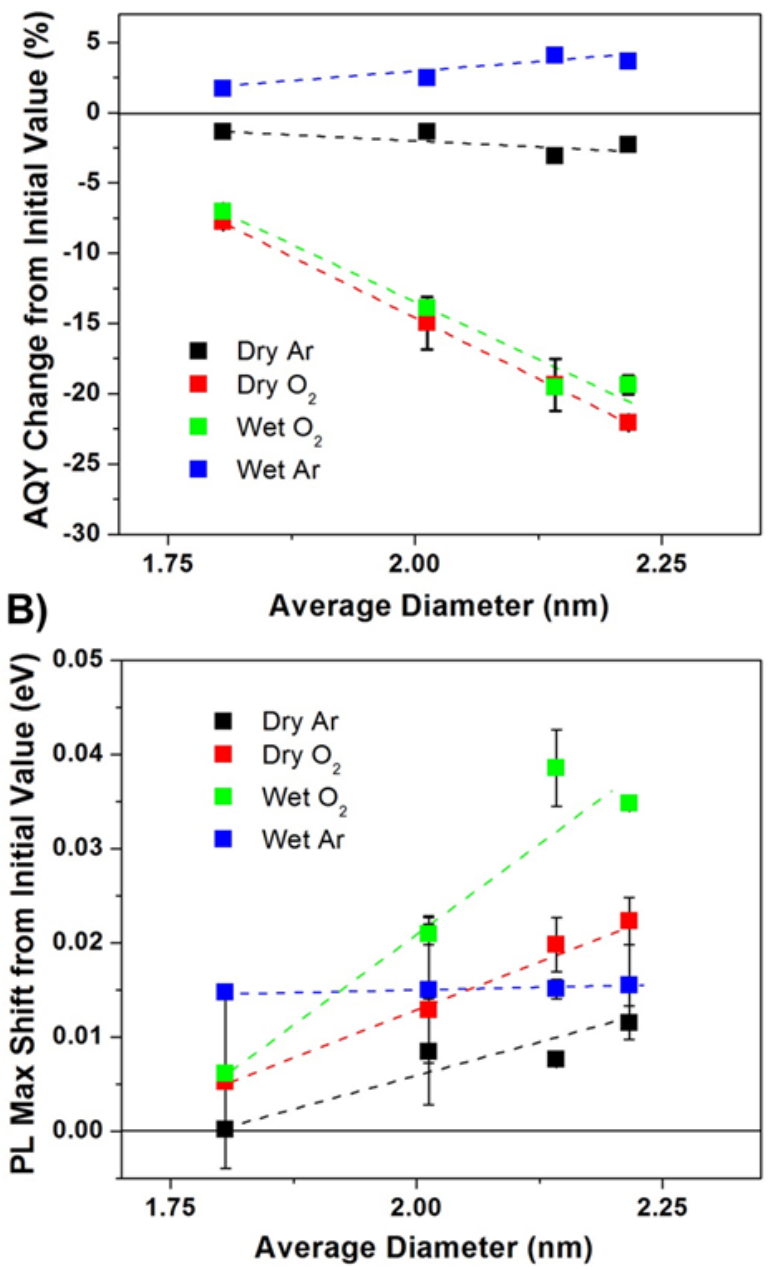

Figure 4. Size-Dependence of AQY and PL Maximum Shifts. A) The value by which the PL AQY changes after exposure to various atmospheres compared to its initial AQY is plotted for several different sized fractions. Dashed lines are shown to visually illustrate the sizedependent trends. B) The value by which the PL maximum shifts after exposure to various atmospheres compared to its initial PL maximum is plotted for several different sized fractions. Dashed lines are shown to visually illustrate the size-dependent trends. In figure A) and B), where error bars are not visible, the associated error value falls within the range of the symbol size. 
- Energy $(E)$ transfer from ncSi:AB to $\mathrm{O}_{2}$ excites it to the singlet state $\left(^{*}\right)$ (photosensitization)

- Excited $\mathrm{O}_{2}$ reacts at the ncSi:AB surface resulting in irreversible surface oxidation

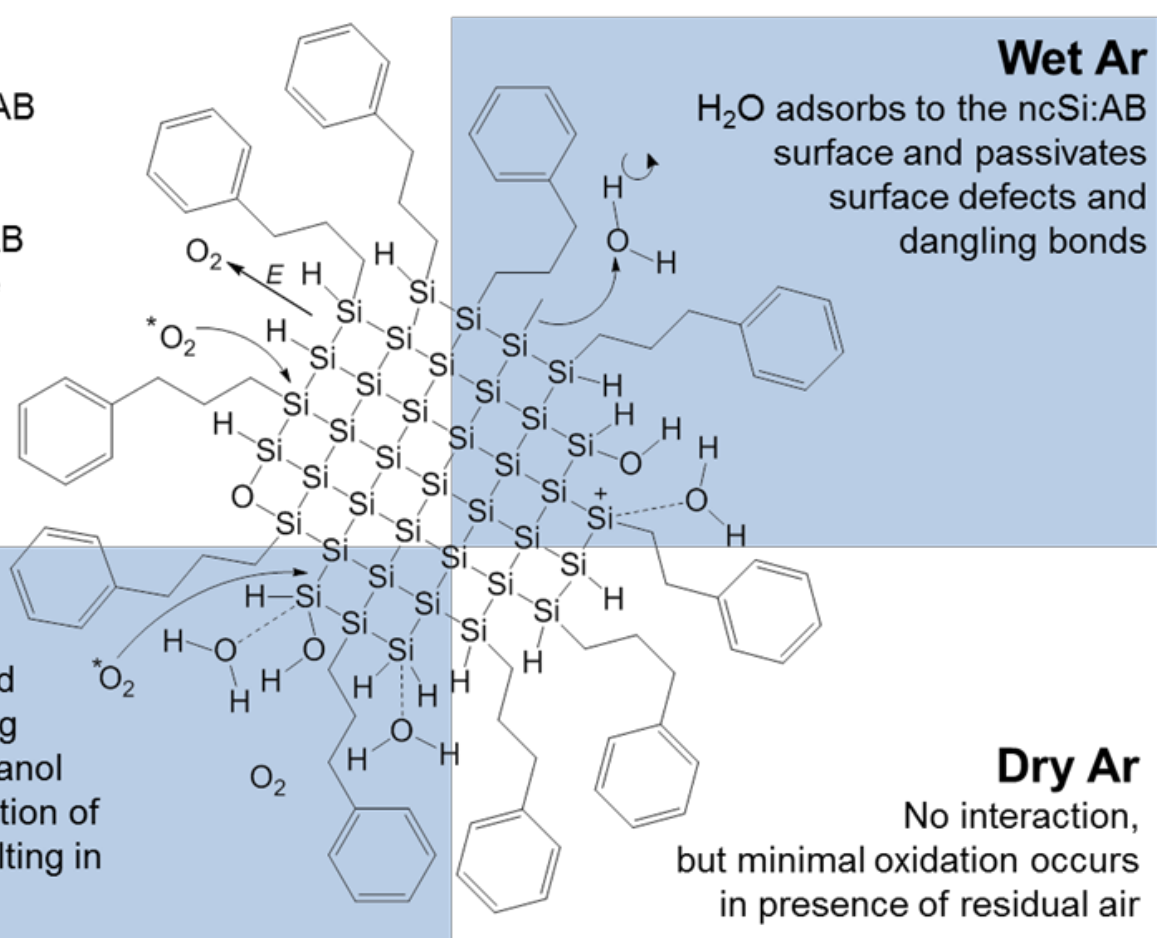

\section{Wet $\mathrm{O}_{2}$}

- $\mathrm{H}_{2} \mathrm{O}$ adsorbs to the ncSi:AB surface passivating defects and preventing $\mathrm{O}_{2}$ from approaching

- $\mathrm{H}_{2} \mathrm{O}$ adsorbed near surface silanol groups helps promote the reaction of $\mathrm{O}_{2}$ at the ncSi:AB surface resulting in irreversible surface oxidation

in presence of residual air

Scheme 1. Summary of proposed interactions occurring at the ncSi:AB surface under each atmosphere.

Continuous AQY curves and PL spectra (similar to those described above for fraction 16) were measured for film samples of four different sized fractions (11, 13, 16, and 18). The difference in PL AQY value (AQY final AQY initial) for each fraction exposed to each atmosphere is plotted in Figure 4A. Similar results as previously outlined for fraction 16 are observed for all sizes examined, where the decrease in AQY is greatest for samples exposed to dry $\mathrm{O}_{2}$, followed by wet $\mathrm{O}_{2}$, then dry $\mathrm{Ar}$, and samples exposed to wet Ar show an increase in AQY. Size-dependent trends can also be extracted from these results, where the AQY shift increases in magnitude as the particle size increases for films exposed to dry $\mathrm{Ar}$, dry $\mathrm{O}_{2}$, and wet $\mathrm{O}_{2}$. Work examining photosensitization of porous silicon suggests that larger particles show the greatest PL degradation due to their larger surface region, which would increase the probability of $\mathrm{O}_{2}$ interacting at the surface. ${ }^{14 c}$ Despite the presence of ligands at the ncSi surface this effect may also occur in our case, such that more PL quenching is possible for the largest particles. This trend may also be explained by the fact that quenching by photosensitization is most efficient when the PL energy occurs at $1.63 \mathrm{eV}(760 \mathrm{~nm}) .{ }^{14 \mathrm{~b}}$ The PL spectra of all fractions have some intensity at this energy but as the particle size decreases the PL maximum blue shifts away from this value so the smaller particles have less overlap with this photosensitization wavelength compared to the largest particles.

In the case where the films are exposed to wet $\mathrm{Ar}$, the magnitude of the AQY increase, increases with increasing size. This trend may also be explained by larger particles having larger surfaces, which likely result in a higher number of surface defects with which $\mathrm{H}_{2} \mathrm{O}$ is able to interact and passivate.

The PL blue shift (final PL maximum - initial PL maximum) in $\mathrm{eV}$ for fractions exposed to all atmospheres
(Figure 4B), shows a size-dependent trend in which a larger PL blue shift is observed for larger particles for all conditions except wet Ar. Since blue shifting of ncSi emission energy has been attributed to shrinking of the nanocrystalline core due to the formation of an oxide layer for ncSi exposed to air overtime,12 it makes sense that the larger particles have the ability to blue shift more than the smaller particles, which contain less core $\mathrm{Si}$. Additionally, it has been reported that particles that have less initial surface oxidation show greater PL blue shifting upon further oxidation, ${ }^{27}$ which fits with the FTIR (Figure 1A) and XPS (SI Figure 3A) measurements of pristine ncSi:AB presented here. XPS measurements of size-separated ncSi:AB after exposure to air overtime also indicate that the atomic \% of oxygen is greatest for larger particles (SI Figure 3B), which is consistent with the observed size-dependent PL blue shifting trends. The PL shift magnitude and slope of each trend follows the order where wet $\mathrm{O}_{2}>$ dry $\mathrm{O}_{2}>$ dry $\mathrm{Ar}$, which is consistent with the results previously explained for fraction 16, where the presence of $\mathrm{H}_{2} \mathrm{O}$ with $\mathrm{O}_{2}$ helps promote the irreversible oxidation of the $\mathrm{ncSi}: \mathrm{AB}$ surface. Under exposure to wet Ar, the interaction of ncSi: $\mathrm{AB}$ with $\mathrm{H}_{2} \mathrm{O}$ has a significant effect on the PL maximum, resulting in a shift of about $0.015 \mathrm{eV}$ for all sizes. While PL shifting is likely to occur from surface oxidation from residual air in the system, the lack of sizedependence observed for wet Ar on the PL maximum of ncSi:AB indicates that there may be size-independent surface effects that are responsible for PL blue shift observed. More extensive study, however, is required to confirm and identify the effects responsible for the observations in wet Ar.

In this study, we have shown that by quantitatively monitoring the effects $\mathrm{O}_{2}, \mathrm{H}_{2} \mathrm{O}$ and $\mathrm{O}_{2}-\mathrm{H}_{2} \mathrm{O}$ environments have on the PL wavelength, intensity and absolute 
quantum yield of ncSi: $\mathrm{AB}$ one can obtain valuable insight into the effect of the size of the silicon core on their chemical reactivity. With the collected results of this investigation it behooves one to think about the molecules and materials effects that most likely contribute to the trends observed for the size-dependent reactivity of ncSi: $\mathrm{AB}$ with $\mathrm{O}_{2}, \mathrm{H}_{2} \mathrm{O}$ and $\mathrm{O}_{2}-\mathrm{H}_{2} \mathrm{O}$.

It is clear from the results presented in this paper that both $\mathrm{O}_{2}$ and $\mathrm{H}_{2} \mathrm{O}$ and mixtures thereof can oxidize the silicon core of ncSi:AB. The contributing effects include size-dependent steric effects relating to the curvature of the silicon core and its effect on packing of covalently bound surface allylbenzene groups, and quantum size effects of the silicon core and how these affect valence and conduction band energies and gaps. The smaller the silicon core with its larger electronic band gap and higher ionization potential, the harder it would be to oxidize with $\mathrm{O}_{2}, \mathrm{H}_{2} \mathrm{O}$ and $\mathrm{O}_{2}-\mathrm{H}_{2} \mathrm{O}$. Additional factors include the surface area of each particle, which relates to the number of surface defects present per ncSi:AB and the number of $\mathrm{H}_{2} \mathrm{O}$ or $\mathrm{O}_{2}$ species that can interact with each particle, as well as the size of the ncSi:AB core, which limits the extent of possible oxidation. Within the framework of this simple model, the surface area, core size and electronic factors favor increased chemical reactivity as the size of $\mathrm{ncSi}: \mathrm{AB}$ increases.

\section{Conclusions}

In this chemical reactivity study, the effect of $\mathrm{O}_{2}, \mathrm{H}_{2} \mathrm{O}$, and a combination of both on the PL and AQY of relatively monodisperse ncSi:AB have been investigated for a range of particle sizes. The dominant interactions thought to be occurring at the $\mathrm{ncSi}: \mathrm{AB}$ surface under each atmosphere are collected together in Scheme 1. To amplify, we have shown that $\mathrm{O}_{2}$ alone is capable of quenching and blue shifting PL, and that PL quenching likely occurs from a combination of reversible photosensitization and irreversible surface oxidation. In the presence of $\mathrm{H}_{2} \mathrm{O}$, the PL quenching that occurs from $\mathrm{O}_{2}$ is minimized, and PL blue shifting is increased, likely due to the passivation of surface defects and dangling bonds by $\mathrm{H}_{2} \mathrm{O}$ to create $\mathrm{Si}-\mathrm{OH}$ species that help promote further irreversible surface oxidation by $\mathrm{O}_{2}$ in the presence of $\mathrm{H}_{2} \mathrm{O}$. Exposure to $\mathrm{H}_{2} \mathrm{O}$ alone results in an increase in the AQY. In all environments, the AQY changes observed increased with increasing particle size, and with the exception of exposure to wet $\mathrm{Ar}$, the PL shifts observed increased with increasing size. Furthermore, in this work we have also shown that the chemical reactivity of the ncSi:AB surface with $\mathrm{H}_{2} \mathrm{O}$ and/ or $\mathrm{O}_{2}$ is increased under continuous illumination with UV light.

\section{ASSOCIATED CONTENT}

Supporting Information. Scanning transmission electron microscopy images and XPS data are available in the Supporting Information. This material is available free of charge via the Internet at http:// pubs.acs.org.

\section{AUTHOR INFORMATION}

\section{Corresponding Author}

*E-mail: gozin@chem.utoronto.ca

\section{ACKNOWLEDGMENT}

The strong and sustained financial support of this research by the Natural Science and Engineering Research Council of Canada (NSERC) is deeply appreciated. A Premier Research Award in support of this research from the Ministry of $\mathrm{Re}$ search Innovation (MRI) Ontario is also gratefully acknowledged. GAO is Government of Canada Tier 1 Canada Research Chair in Materials Chemistry. MLM is a Vanier Canada Graduate Scholar. This research used equipment funded by the Australian Research Council (ARC) - Linkage, Infrastructure, Equipment and Facilities (LIEF) grant (LE120100104) located at the UOW Electron Microscopy Centre. Dr. Peter Brodersen and Surface Interface Ontario are thanked for XPS measurements.

\section{REFERENCES}

(1) Canham, L. T. Appl. Phys. Lett. 1990, 57, 1046.

(2) (a) Mastronardi, M. L.; Maier-Flaig, F.; Faulkner, D.; Henderson, E. J.; Kübel, C.; Lemmer, U.; Ozin, G. A. Nano Lett. 2012, 12, 337. (b) Mastronardi, M. L. M.; Hennrich, F.; Henderson, E. J.; Maier-Flaig, F.; Blum, C.; Reichenbach, J .; Lemmer, U.; Kübel, C.; Wang, D.; Kappes, M. M.; Ozin, G. A. J . Am. Chem. Soc. 2011, 133, 11928. (c) Hessel, C. M.; Reid, D.; Panthani, M. G.; Rasch, M. R.; Goodfellow, B. W.; Wei, J.; Fujii, H.; Akhavan, V.; Korgel, B. A. Chem. Mater. 2012, 24, 393.

(3) Ledoux, G.; Guillois, O.; Porterat, D.; Reynaud, C.; Huisken, F.; Kohn, B.; Paillard, V. Phys. Rev. B 2000, 62, 15942.

(4) (a) Miller, J. B.; Van Sickle, A. R.; Anthony, R. J.; Kroll, D. M.; Kortshagen, U. R.; Hobbie, E. K. ACS Nano 2012, 6, 7389. (b) Rinck, J.; Schray, D.; Kübel, C.; Powell, A. K.; Ozin, G. A. Small 2014, 1

(5) (a) Wu, C.; Bull, B.; Szymanski, C.; Christensen, K.; McNeill, J. ACS Nano 2008, 2, 2415. (b) Henderson, E. J.; Shuhendler, A. J.; Prasad, P.; Baumann, V.; Maier-Flaig, F.; Faulkner, D. O.; Lemmer, U.; Wu, X. Y.; Ozin, G. A. Small 2011, 7, 2507. (c) Mastronardi, M. L.; Henderson, E. J.; Puzzo, D. P.; Chang, Y.; Wang, Z. Bin; Helander, M. G.; J eong, J.; Kherani, N. P.; Lu, Z.; Ozin, G. A. Small 2012, 8, 3647. (d) Cheng, K.-Y.; Anthony, R.; Kortshagen, U. R.; Holmes, R. J. Nano Lett. 2011, 11, 1952. (e) Cheng, K.-Y.; Anthony, R.; Kortshagen, U. R.; Holmes, R. J. Nano Lett. 2010, 10, 1154.

(6) Liu, S.-M.; Sato, S.; Kimura, K. Chem. Mater. 2006, 18, 637.

(7) Pi, X. D.; Liptak, R. W.; Deneen Nowak, J.; Wells, N. P.; Carter, C. B.; Campbell, S. A; Kortshagen, U. Nanotechnology 2008, 19, 245603.

(8) Zou, J.; Sanelle, P.; Pettigrew, K. A.; Kauzlarich, S. M. J. Clust. Sci. 2006, 17, 565.

(9) (a) Buriak, J. Chem. Mater. 2013, 26, 763. (b) Kelly, J . A; Shukaliak, A. M.; Fleischauer, M. D.; Veinot, J. G. C. J. Am. Chem. Soc. 2011, 133, 9564. (c) Kelly, J . A; Veinot, J . G. C. ACS Nano 2010, 4, 4645.

(10) Pi, X.; Wang, R.; Yang, D. J. Mater. Sci. Technol. 2014, 30, 639.

(11) Jurbergs, D.; Rogojina, E.; Mangolini, L.; Kortshagen, U. Appl. Phys. Lett. 2006, 88, 233116.

(12) Anthony, R.; Kortshagen, U. Phys. Rev. B 2009, 80, 115407.

(13) (a) Pereira, R. N.; Rowe, D. J.; Anthony, R. J .; Kortshagen, U. Phys. Rev. B 2011, 83, 155327. (b) Delley, B.; Steigmeier, E. Phys. Rev. B 1993, 47, 1397.

(14) (a) Konstantinova, E. A.; Demin, V. A.; Vorontzov, A. S.; Ryabchikov, Y. V.; Belogorokhov, I. A.; Osminkina, L. A.; Forsh, P. A.; Kashkarov, P. K.; Timoshenko, V. Y. J. Non. Cryst. Solids 2006, 352, 1156. (b) Kovalev, D.; Gross, E.; Künzner, N.; Koch F.; Timoshenko, V.; Fujii, M. Phys. Rev. Lett. 2002, 89, 137401. (c) Kovalev, D.; Gross, E.; Diener, J.; Timoshenko, V. Y.; Fujii, M. Appl. Phys. Lett. 2004, 85, 3590. 
(15) (a) Miura, T.; Niwano, M.; Shoji, D.; Miyamoto, N. J . Appl. Phys. 1996, 79, 4373. (b) Niwano, M.; Kageyama, J.; Kurita, K. J . Appl. Phys. 1994, 76, 2157.

(16) (a) Švrček, V.; Sasaki, T.; Shimizu, Y.; Koshizaki, N. Appl. Phys. Lett. 2006, 89, 213113. (b) Li, S.; Silvers, S. J.; Elshall, M. S.; Commonwealth, V.; Uni, V. 2006, 5647, 1794.

(17) Stewart, M. P.; Buriak, J. M. J. Am. Chem. Soc. 2001, 123, 7821.

(18) Panthani, M.; Hessel, C.; Reid, D.; Casillas, G.; J oseYacaman, M.; Korgel, B. A. J . Phys. Chem. C 2012, 116, 22463.

(19) Mangolini, L.; J urbergs, D.; Rogojina, E.; Kortshagen, U. J . Lumin. 2006, 121, 327.

(20) Faulkner, D. O.; McDowell, J . J .; Price, A. J .; Perovic, D. D.; Kherani, N. P.; Ozin, G. A. Laser Photon. Rev. 2012, 6, 802. (21) Lopinski, G.; Wayner, D. Mater. Matters 2008, 3, 38.

(22) Bullen, C.; Mulvaney, P. Langmuir 2006, 22, 3007.
(23) Hessel, C. M.; Rasch, M. R.; Hueso, J . L.; Goodfellow, B. W.; Akhavan, V. A; Puvanakrishnan, P.; Tunnel, J. W.; Korgel, B. A. Small 2010, 6, 2026.

(24) Mei, B. C.; Oh, E.; Susumu, K.; Farrell, D.; Mountziaris, T. J .; Mattoussi, H. Langmuir 2009, 25, 10604.

(25) (a) Lockwood, R.; Hryciw, A.; Meldrum, A. Appl. Phys. Lett. 2006, 89, 263112. (b) Priolo, F.; Franzò, G.; Pacifici, D.; Vinciguerra, V.; Iacona, F.; Irrera, A. J . Appl. Phys. 2001, 89, 264.

(26) Yang, J .; Liptak, R.; Rowe, D.; Wu, J .; Casey, J .; Witker, D.; Campbell, S. A.; Kortshagen, U. Appl. Surf. Sci. 2014.

(27) Froner, E.; Adamo, R.; Gaburro, Z.; Margesin, B.; Pavesi, L.; Rigo, A.; Scarpa, M. J. Nanoparticle Res. 2006, 8, 1071. 
SYNOPSIS TOC:

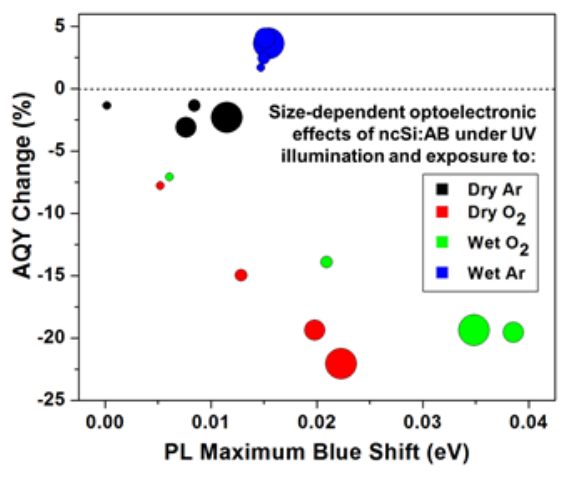




\section{Size-Dependant Chemical Reactivity of Silicon Nanocrystals}

Melanie L. Mastronardi, ${ }^{\dagger}$ Kenneth K. Chen, ${ }^{\dagger}$ Kristine Liao, ${ }^{\dagger}$ Gilberto Casillas, ${ }^{\alpha}$ Geoffrey A. Ozin ${ }^{\dagger} *$

${ }^{\dagger}$ Department of Chemistry, University of Toronto, 80 St. George Street, Toronto, Ontario, Canada, M5S 3H6

${ }^{\alpha}$ UOW Electron Microscopy Centre, University of Wollongong, New South Wales 2500, Australia

* To whom correspondence should be addressed: gozin@chem.utoronto.ca
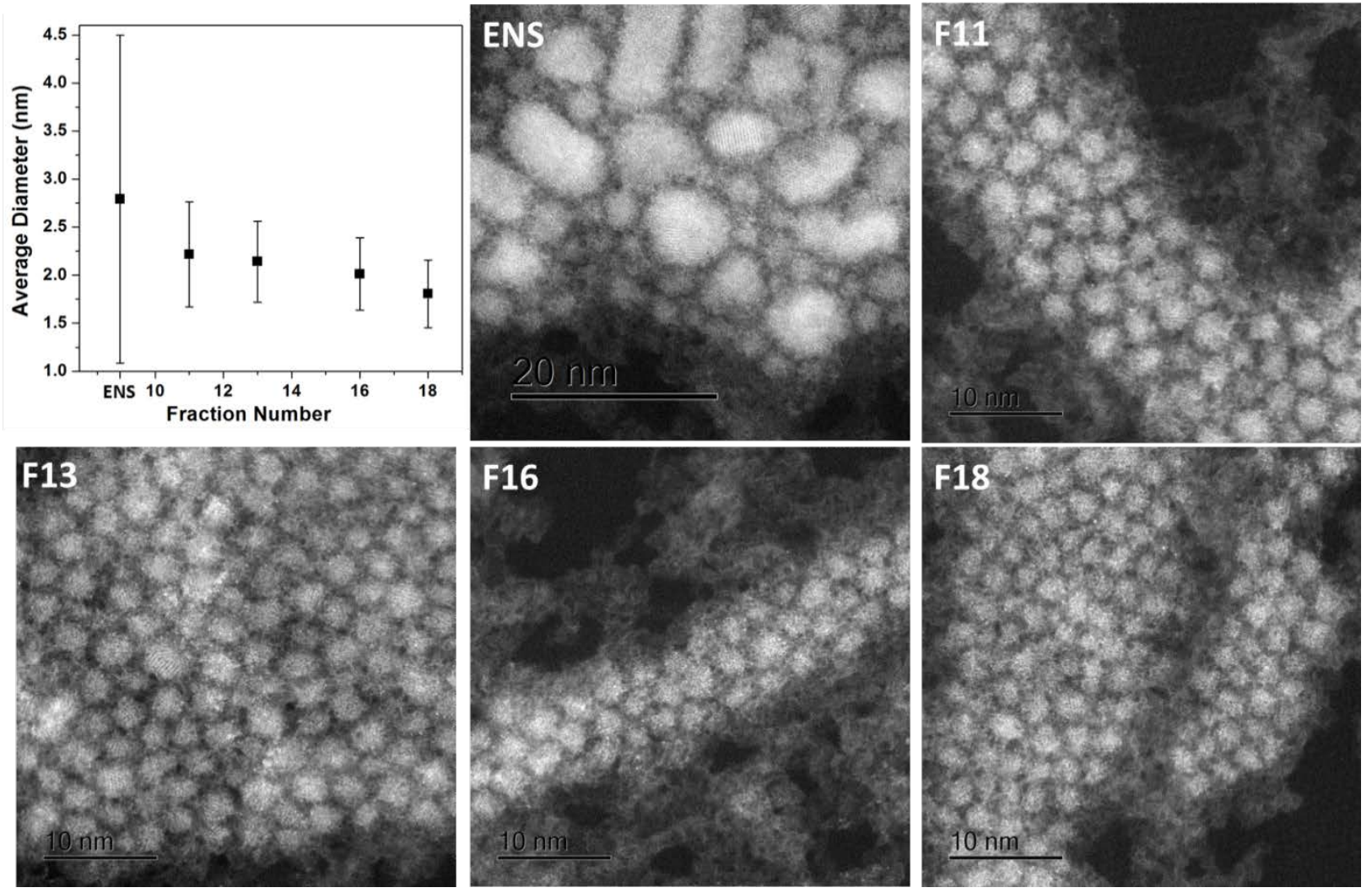

SI Figure 1. Middle Angle Annular Dark Field Scanning Transmission Electron Microscopy. Representative images of the polydisperse ensemble and select size-separated ncSi:AB fractions. 

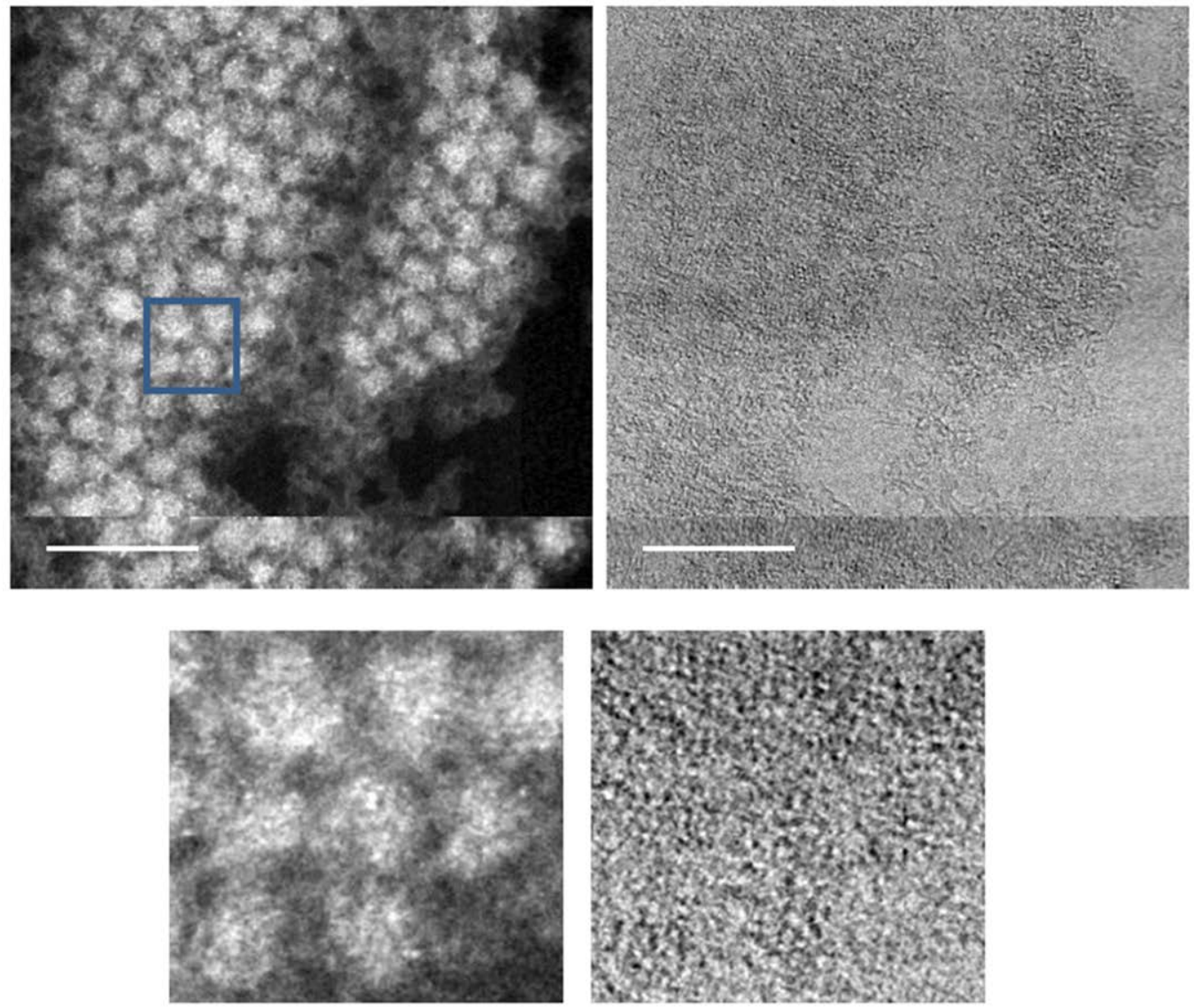

SI Figure 2. Scanning Transmission Electron Microscopy of Fraction 18. Top images show the same area measured under middle angle annular dark field (left) and bright field (right). Bottom images are a magnification of the area denoted by the blue square. Scale bar indicates $10 \mathrm{~nm}$. 


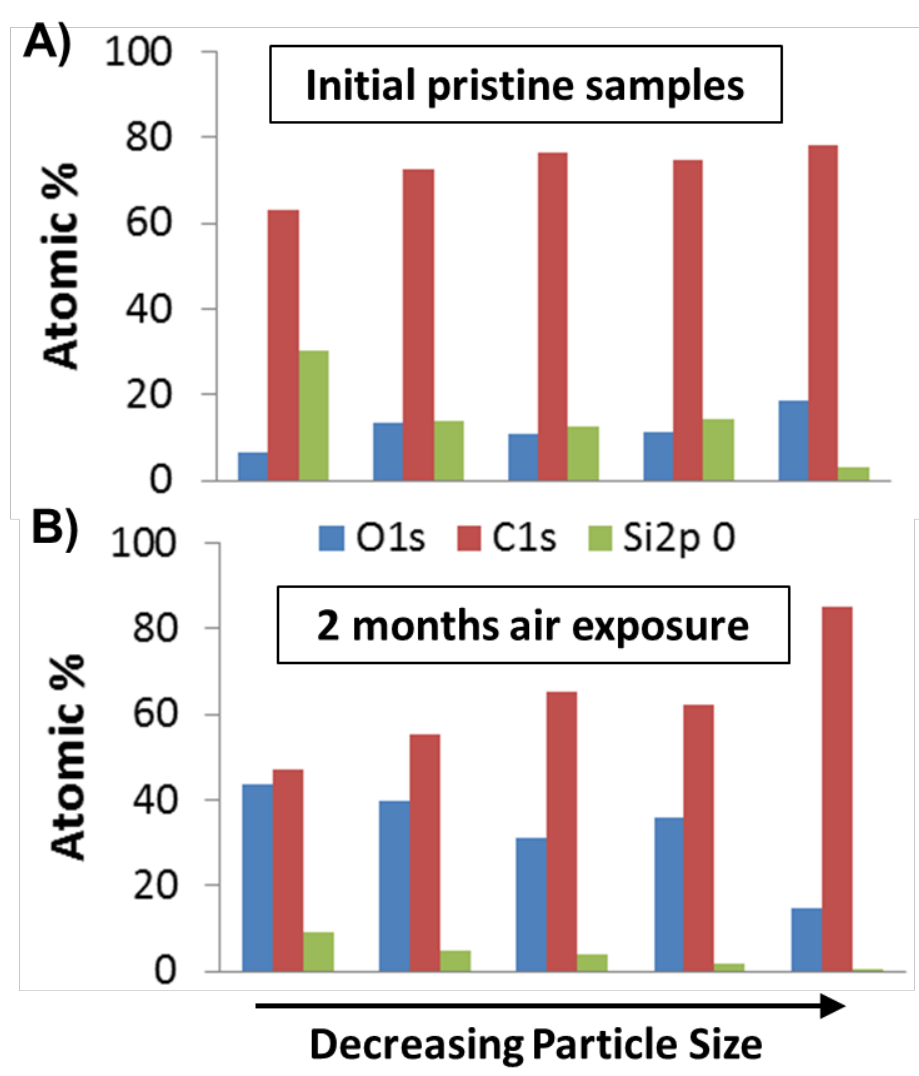

SI Figure 3. Atomic percent from X-ray photoelectron spectroscopy (XPS) of size-separated ncSi:AB. Atomic \% of $\mathrm{O} 1 \mathrm{~s}, \mathrm{C} 1 \mathrm{~s}$ and $\mathrm{Si} 2 \mathrm{p}^{0}$ species as determined by XPS for size-separated ncSi:AB of varying size immediately after synthesis (A), and after exposure to air for about 2 months (B). 
- Energy $(E)$ transfer from ncSi:AB to $\mathrm{O}_{2}$ excites it to the singlet state $\left({ }^{*}\right)$ (photosensitization)

- Excited $\mathrm{O}_{2}$ reacts at the ncSi:AB surface resulting in irreversible surface oxidation

\section{Wet $\mathrm{O}_{2}$}

- $\mathrm{H}_{2} \mathrm{O}$ adsorbs to the ncSi:AB surface passivating defects and preventing $\mathrm{O}_{2}$ from approaching

- $\mathrm{H}_{2} \mathrm{O}$ adsorbed near surface silanol groups helps promote the reaction of $\mathrm{O}_{2}$ at the ncSi:AB surface resulting in irreversible

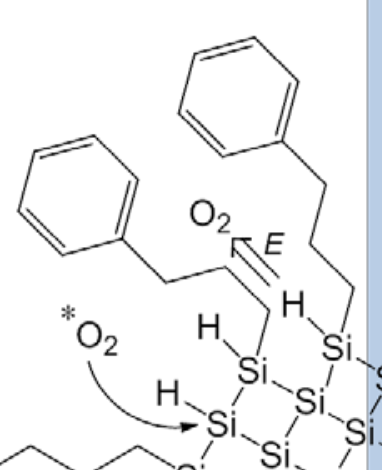

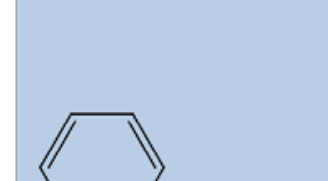

Wet Ar $\mathrm{H}_{2} \mathrm{O}$ adsorbs to the ncSi:AB surface and passivates surface defects and dangling bonds surface oxidation

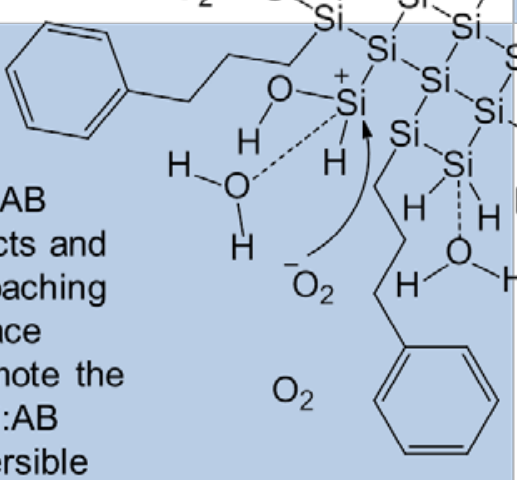

$\mathrm{O}_{2}$

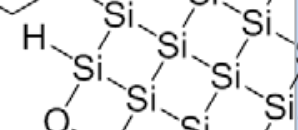

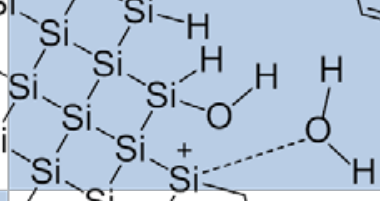<smiles>C1CCC1</smiles><smiles>CCC[Si]1(CCCc2ccccc2)C[SiH]2C[SiH]1[SiH]2CC</smiles><smiles>CCC[Si]1(C)[SiH2][Si](C)(C)[Si]2([SiH2])[SiH2]C[SiH]12</smiles>

No interaction, but minimal oxidation occurs in presence of residual air

SI Figure 4. Summary of proposed interactions occurring at the ncSi:AB surface under each atmosphere. 